\title{
APPROXIMATION AND BESOV SPACES ON STRATIFIED GROUPS
}

\author{
SAVERIO GIULINI
}

\begin{abstract}
This paper treats analogues of the classical Bernstein and Jackson theorems in the nonclassical context of the stratified groups. Besov spaces on stratified groups are characterized by their approximation theoretic properties.
\end{abstract}

Let $G$ be a stratified group. If $\mathscr{L}=-\sum_{j=0}^{n} X_{j}^{2}$ is the sublaplacian of $G$ and $\mathscr{L}=\int_{0}^{+\infty} \lambda d E(\lambda)$ is its spectral resolution, we define $\mathscr{M}_{p}(h)=\left\{f \in L^{p}: E(\lambda) f=f\right.$ if $\left.\lambda \geqslant h^{2}\right\}(1 \leqslant p \leqslant+\infty, h \in[0,+\infty))$. This class of functions in $G$ plays a role analogous to entire functions of exponential type in $\mathbf{R}^{n}$. In fact we prove Jackson's theorem about the best approximation $E_{p}(f, h)=\operatorname{Inf}_{g \in \mathscr{M}_{p}(h)}\|f-g\|_{p}$ of an $L^{p}$ function by means of functions in $\mathscr{M}_{p}(h)$. Moreover, Bernstein's theorem holds for the class $\mathscr{M}_{p}(h)$ in a slightly modified form. $\S 3$ is devoted to the proofs of these theorems after some preliminary properties on moduli of smoothness which are proved in $\S 2$. Finally, in $\S 4$ we give a characterization of the functions of the Besov space $B_{p, q}^{s}(G)$ by the behavior of the best approximations $E_{p}(h, f)$.

We are happy to thank Giancarlo Mauceri for some helpful observations and suggestions. We are also indebted to the referee who brought Saka's paper [10] to our attention.

1. The real finite dimensional Lie algebra $\mathrm{g}$ is called stratified if $\mathrm{g}=\sum_{k=1}^{m} \mathscr{V}_{k}$ as a vector space, where $\mathscr{V}_{1}$ generates $g$ as an algebra, $\left[\mathscr{V}_{1}, \mathscr{V}_{k}\right]=\mathscr{V}_{k+1}(k=1, \ldots, m$ $-1)$ and $\left[\mathscr{V}_{1}, \mathscr{V}_{m}\right]=0$. We can define in $g$ a natural family of dilations

$$
\delta_{r}\left(\sum_{k=1}^{m} X_{k}\right)=\sum_{k=1}^{m} r^{k} X_{k} \quad\left(X_{k} \in \mathscr{V}_{k}, r>0\right) .
$$

A stratified group is a connected, simply connected, nilpotent Lie group $G$ whose Lie algebra $\mathfrak{g}$ is stratified. Then the exponential map is a diffeomorphism of $\mathfrak{g}$ onto $G$, and we can transfer, via the exponential map, the dilations $\delta_{r}$ to $G$. We can define on $G$ a homogeneous norm; i.e., a function $x \mu|x|$ from $G$ to $[0,+\infty)$, which is $\mathscr{C}^{\infty}$ on $G-\{e\}$ and satisfies (a) $\left|x^{-1}\right|=|x|$, (b) $\left|\delta_{r} x\right|=r|x|$, and (c) $|x|=0$ if and only if $x=e$, the origin of $G$. The number $Q \mp \sum_{k=1}^{m} k \operatorname{dim} \mathscr{V}_{k}$ is called the homogeneous dimension of $G$, and we define

$$
f_{(r)}=r^{-Q} f \circ \delta_{1 / r} \quad(r>0) .
$$

Received by the editors January 17, 1985.

1980 Mathematics Subject Classification. Primary 41A17, 43A80.

Key words and phrases. Bernstein and Jackson type inequalities, stratified groups, Besov spaces. 
We fix a basis $X_{1}, \ldots, X_{s}$ for $g$ and suppose that $X_{1}, \ldots, X_{n}$ is a basis for $\mathscr{V}_{1}$. The first order differential operators

$$
X_{k} f(x)=\left.\frac{d}{d t}\right|_{t=0} f\left(x \exp \left(t X_{k}\right)\right) \quad\left(f \in \mathscr{C}^{1}\right),
$$

corresponding to the element $X_{k} \in \mathscr{V}_{j}(k=1, \ldots, s ; j=1, \ldots, m)$, are homogeneous of degree $j$ with respect to the dilations $\delta_{r}$ :

$$
X_{k} f_{(r)}=r^{-j}\left(X_{k} f\right)_{(r)} .
$$

We denote by $\mathscr{D}_{s}$ the set of all the differential monomials

$$
X_{j_{1}}^{i_{1}} \cdots X_{j_{k}}^{i_{k}}
$$

where $i_{1}, \ldots, i_{k}$ are nonnegative integers such that $i_{1}+\cdots+i_{k}=s$ and $j_{1}, \ldots, j_{k}$ $\in\{1, \ldots, n\}$. The set $\mathscr{D}_{s}$ spans the vector space of left invariant differential operators which have homogeneous degree $s$. The sublaplacian of $G$ is the left invariant differential operator, homogeneous of degree $2, \mathscr{L}=-\sum_{k=1}^{n} X_{k}^{2}$. We remark that the operators $X_{k}$ are formally skew-adjoint. Moreover,

$$
X_{k}(f * g)=f *\left(X_{k} g\right), \quad f, g \in \mathscr{C}^{\infty}(G) .
$$

The image under the exponential map of Lebesgue measure on $\mathfrak{g}$ is a (bi-invariant) Haar measure $d x$ on $G$. We have $d\left(\delta_{r} x\right)=r^{Q} d x$.

2. Let $f$ be a locally integrable function. For every integer $N$ we define

$$
\Delta_{x}^{N} f(y)=\sum_{j=0}^{N}(-1)^{N+j}\left(\begin{array}{l}
N \\
j
\end{array}\right) f\left(y \delta_{j} x\right) \quad(x, y \in G) .
$$

If $h \in \mathbf{R}^{+}$and $N \in \mathbf{N}$, we define the $N$ th modulus of smoothness

$$
\omega_{N}(h, f)=\omega_{N}\left(h, L^{p}, f\right)=\sup _{|x|<h}\left\|\Delta_{x}^{N} f\right\|_{p} \quad(1 \leqslant p \leqslant \infty) .
$$

Moduli of smoothness in the Heisenberg group $H_{n}$ were studied by I. R. Inglis [6] and by the author [5], and by P. M. Soardi [11, 12] in the nonisotropic $\mathbf{R}^{n}$ and in the general homogeneous group.

We denote by $L_{s}^{p}$ the space of all functions $f$ such that $f$ and its distributional derivatives $D f\left(D \in \mathscr{D}_{k}, k \leqslant s\right)$ all belong to $L^{p}$. We define $\left\|f^{(s)}\right\|_{p}=\sum_{D \in \mathscr{D}_{s}}\|D f\|_{p}$; $\omega_{N}\left(h, f^{(s)}\right)$ has an analogous meaning. From now on $\|\cdot\|$ shall denote $\|\cdot\|_{p}$, unless otherwise stated.

Proposition 1. Let $N$ be a positive integer. There exists a constant $C$ such that

$$
\left\|\Delta_{x}^{N} g\right\| \leqslant c|x|^{N}\left\|g^{(N)}\right\|
$$

for every $g$ in $L_{N}^{p}(1 \leqslant p \leqslant \infty)$.

Proof. There exists an integer $M$ such that every $x \in G$ can be written $x=x_{1}$ $\cdots x_{M}$, where $x_{j}=\exp X^{(j)}$ with $X^{(j)} \in \mathscr{V}_{1}$ and $\left|x_{j}\right| \leqslant C|x|$ (see [4, 1.40]). Then we have

$$
\Delta_{x}^{N} g(y)=\sum_{k=1}^{M-1}\left(\Delta_{x_{1} \cdots x_{k+1}}^{N} g(y)-\Delta_{x_{1} \cdots x_{k}}^{N} g(y)\right)+\Delta_{x_{1}}^{N} g(y) .
$$


Obviously

$$
\begin{aligned}
& g\left(y \delta_{j}\left(x_{1} \cdots x_{k}\right)\right)-g\left(y \delta_{j}\left(x_{1} \cdots x_{k-1}\right)\right) \\
& \quad=\int_{0}^{j} \frac{d}{d t} g\left(y \delta_{j}\left(x_{1} \cdots x_{k-1}\right) \exp t X^{(k)}\right) d t \\
& =\int_{0}^{j} X^{(k)} g\left(y \delta_{j}\left(x_{1} \cdots x_{k-1}\right) \exp t X^{(k)}\right) d t .
\end{aligned}
$$

If we set $X^{(k)}=\sum_{r=1}^{n} c_{r}^{(k)} X_{r}$, where $\left|c_{r}^{(k)}\right| \leqslant\left|\exp X^{(k)}\right| \leqslant C|x|(k=1, \ldots, M ; r=$ $1, \ldots, n)$,

$$
\begin{aligned}
& \Delta_{x_{1} \cdots x_{k}}^{N} g(y)-\Delta_{x_{1} \cdots x_{k-1}}^{N} g(y) \\
& \quad=\sum_{r=1}^{n} c_{r}^{(k)} \int_{0}^{1} \sum_{j=1}^{N}(-1)^{j}\left(\begin{array}{c}
N \\
j
\end{array}\right) j X_{r} g\left(y \delta_{j}\left(x_{1} \cdots x_{k-1}\right) \exp j t X^{(k)}\right) d t \\
& \quad=\sum_{r=1}^{n} c_{r}^{(k)} \int_{0}^{1} \Delta_{x_{1} \cdots x_{k-1} \exp t X^{(k)}}^{N-1} X_{r} g(y) d t
\end{aligned}
$$

There exists a constant $\gamma$ such that $\left|x_{1} \cdots x_{k} \exp t X^{(k)}\right| \leqslant \gamma|x|(k=1, \ldots, M)$. Since $\omega(\cdot, g)$ is an increasing function, we obtain

$$
\begin{aligned}
\left\|\Delta_{x_{1} \cdots x_{k}}^{N} g-\Delta_{x_{1} \cdots x_{k-1}}^{N} g\right\| & \leqslant C|x| \sum_{r=1}^{n} \omega_{N-1}\left(\gamma|x|, X_{r} g\right) \\
& =C|x| \omega_{N-1}\left(\gamma|x|, g^{(1)}\right) .
\end{aligned}
$$

We can estimate $\left\|\Delta_{x_{1}}^{N} g(y)\right\|$ in a similar way since

$$
\Delta_{x_{1}}^{N} g(y)=\sum_{j=1}^{N}(-1)^{j+1}\left(\begin{array}{l}
N \\
j
\end{array}\right)\left(g\left(y \delta_{j}\left(x_{1}\right)\right)-\xi(y)\right) .
$$

Hence

$$
\left\|\Delta_{x}^{N} g\right\| \leqslant C|x| \omega_{N-1}\left(\gamma|x|, g^{(1)}\right) .
$$

By iteration we get

$$
\begin{aligned}
\left\|\Delta_{x}^{N} g\right\| & \leqslant C^{k} \gamma^{k(k-1) / 2}|x|^{k} \omega_{N-k}\left(\gamma^{k}|x|, g^{(k)}\right) \quad(0<k<N) \\
& \leqslant C^{\prime}|x|^{N}\left\|g^{(N)}\right\| .
\end{aligned}
$$

Corollary 1. Let $N$ be an integer. There exist two constants $C_{1}$ and $C_{2}$ such that

$$
C_{1} \omega_{N}(h, f) \leqslant \operatorname{Inf}_{g \in L_{N}}\left(\|f-g\|+h^{N}\left\|g^{(N)}\right\|\right) \leqslant C_{2} \omega_{N}(h, f)
$$

for every $f \in L^{p}(1 \leqslant p \leqslant \infty)$ and $h>0$.

Proof. For the right side of (4) we can repeat verbatim the proof of the corresponding inequality in $H_{n}\left[\mathbf{5}\right.$, Lemma 2] or in nonisotropic $\mathbf{R}^{n}$ [11, Theorem 4]. On the other hand,

$$
\left\|\Delta_{x}^{N} f\right\| \leqslant\left\|\Delta_{x}^{N}(f-g)\right\|+\left\|\Delta_{x}^{N} g\right\|<2^{N}\|f-g\|+\left\|\Delta_{x}^{N} g\right\| .
$$

The assertion follows by Proposition 1 . 
COROLlary 2. Let $N$ be a positive integer. There exists a constant $C$ such that

$$
\omega_{N}(\lambda h, f) \leqslant C\left(1+\lambda^{N}\right) \omega_{N}(h, f)
$$

for every $\lambda$ and $h>0$ and $f \in L^{p}$.

Corollary 3. Let $N$ and $k$ be two integers such that $N \geqslant k \geqslant 0$. There exists a constant $C$ such that

$$
\left\|\Delta_{x}^{N} g\right\| \leqslant C|x|^{k} \omega_{N-k}\left(|x|, g^{(k)}\right)
$$

for every $g \in L_{k}^{p}$.

Proof. The proof follows by (3) and Corollary 2.

3. Let $\mathscr{L}=\int_{0}^{+\infty} \lambda d E(\lambda)$ be the spectral resolution of the subLaplacian. If $\phi$ is a bounded Borel function on $\mathbf{R}^{+}$, we define the bounded operator on $L^{2}$,

$$
\phi(\mathscr{L})=\int_{0}^{+\infty} \phi(\lambda) d E(\lambda) .
$$

By the Schwartz kernel theorem $\phi(\mathscr{L})$ has a tempered distribution kernel $K$ such that $\phi(\mathscr{L}) f=f * K$ for every $f \in \mathscr{S}(G)$, the Schwartz space of $G$. The kernel distribution of the operator $\phi(t \mathscr{L})=\int_{0}^{+\infty} \phi(t \lambda) d E(\lambda)$ is

$$
K_{(\sqrt{t})}(x)=t^{-Q / 2} K\left(\delta_{t^{-1 / 2}} x\right)
$$

(see $[4,6.29])$.

In a recent work $G$. Mauceri [8] proved, among other things, the following result, which is a consequence of Lemma 6.36 in [4].

Theorem (G. MAUCERI [8, Proposition 2.7]). Let $\phi$ be the restriction to $\mathbf{R}^{+}$of a function in the Schwartz space $\mathscr{S}(\mathbf{R})$. Then the kernel $K$ of the operator $\phi(\mathscr{L})$ is in the Schwartz space $\mathscr{S}(G)$.

Definition. Let $h$ be a positive number. We denote by $\mathscr{M}_{p}(h)(1 \leqslant p \leqslant \infty)$ the space of all the functions $f \in L^{p}$ such that

$$
E(\lambda) f=f \quad \text { if } \lambda \geqslant h^{2} .
$$

The class $\mathscr{M}_{p}(h)$ plays in $G$ the same role as that of trigonometric polynomials of degree less than $h$ in $\mathbf{T}^{n}$ or that of the entire functions of exponential type $h$ in $\mathbf{R}^{n}$. The following theorems are the versions of classical Bernstein and Jackson theorems for stratified groups.

BERNSTEIN'S THEOREM. Let $D$ be a left invariant differential operator homogeneous of degree s. There exists a constant $C=C(D)$ such that $\|D g\| \leqslant C h^{s}\|g\|$ for every $g \in \mathscr{M}_{p}(h)$.

Proof. Let $\phi \in \mathscr{C}_{c}^{\infty}\left(\mathbf{R}^{+}\right)$such that $\phi(\xi)=1$ if $\xi \in[0,1]$. If $g \in \mathscr{M}_{p}(h)$, then $\phi\left(h^{-2} \mathscr{L}\right) g=\phi\left(h^{-2} \mathscr{L}\right) E\left(h^{2}\right) g=g$. Therefore if $K$ is the kernel of the operator $\phi(\mathscr{L})$, then $g=g * K_{(1 / h)}$ is a $\mathscr{C}^{\infty}$ function. By (1) and (2) we obtain

$$
\|D g\|=\left\|g * D\left(K_{(1 / h)}\right)\right\|=h^{s}\left\|g *(D K)_{(1 / h)}\right\| \leqslant C h^{s}\|g\| .
$$


REMARK. If we consider $G=H_{n}$, the Heisenberg group, the left invariant vector fields $Z_{j}$ and $\bar{Z}_{j}$ behave, in terms of the Fourier transform, as weighted shifts. As a consequence it is easy to prove that $C\left(Z_{j}^{s}\right) \rightarrow \infty$ if $s \rightarrow \infty$ (for details see [5, remark after Theorem 2]).

JACKSON'S THEOREM. Let $N$ be a positive integer. For every function $f \in L^{p}$ and every positive integer $h$, there exists a function $g_{h} \in \mathscr{M}_{p}(h)$ such that

$$
\left\|f-g_{h}\right\| \leqslant C(N) \omega_{N}(1 / h, f),
$$

where $C(N)$ is a constant which depends only on $N$.

Proof. Let $\phi \in \mathscr{C}_{c}^{\infty}\left(\mathbf{R}^{+}\right)$be a function such that $\operatorname{supp} \phi \subset[0,1]$ and $\phi(0)=1$. The corresponding operator $\phi(\mathscr{L})$ has a Schwartz kernel such that $\int_{G} K(x) d x=1$ (it follows by the spectral theorem and 1.20 of [4]). Let

$$
H=\sum_{j=1}^{N}(-1)^{N+j}\left(\begin{array}{c}
N \\
j
\end{array}\right) K_{(j / h)}
$$

Now if $\delta$ is the Dirac measure supported at $e$,

$$
E(\lambda) H=\sum_{j=1}^{N}(-1)^{N+j}\left(\begin{array}{l}
N \\
j
\end{array}\right) E(\lambda) \phi\left(\left(\frac{h}{j}\right)^{2} \mathscr{L}\right) \delta=H
$$

if $\lambda \geqslant h^{2}$. Hence, $H \in \mathscr{M}_{p}(h)$ and $\int_{G} H(x) d x=(-1)^{N+1}$. We set $g_{h}=f * H$. Hence,

$$
\begin{aligned}
g_{h}(x)-f(x) & =\int_{G}\left(f\left(x y^{-1}\right)+(-1)^{N} f(x)\right) \sum_{j=1}^{N}(-1)^{N+j} K_{(j / h)}(y)\left(\begin{array}{c}
N \\
j
\end{array}\right) d v \\
& =\int_{G} K(v) \sum_{j=0}^{N}(-1)^{N+j} f\left(x \delta_{j / h} v^{-1}\right)\left(\begin{array}{c}
N \\
j
\end{array}\right) d v \\
& =\int_{G} K(v) \Delta_{\delta_{j / h} v^{-1}} f(x) d v
\end{aligned}
$$

The assertion follows by Corollary 2:

$$
\left\|g_{h}-f\right\| \leqslant C(N) \omega_{N}\left(\frac{1}{h}, f\right) \int_{G} K(v)\left(1+|v|^{N}\right) d v .
$$

4. Besov spaces in stratified groups were widely studied by K. Saka in [10]. Following Stein's approach, Saka defines these spaces by using the heat semigroup. For our purpose it is worthwhile to provide a different definition of Besov spaces. However a comparison between Theorems 12 of [10] and Proposition 4 below reveals that the spaces $\Lambda(\alpha, p, q)$ defined by Saka coincide with our spaces $B_{p, q}^{\alpha}$. We recall that the case of the Lipschitz spaces $(q=\infty)$ in stratified groups have been studied in great detail by S. G. Krantz in [7] (see also [3]). 
Let $\phi \in \mathscr{C}_{c}^{\infty}\left(\mathbf{R}^{+}\right)$such that $\operatorname{supp} \phi \subset\left[\frac{1}{4}, 4\right]$ and $\sum_{k=-\infty}^{+\infty} \phi\left(2^{-2 k} \xi\right)=1$ if $\xi>0$. We set

$$
\phi_{0}(\xi)= \begin{cases}\sum_{k=-\infty}^{0} \phi\left(2^{-2 k} \xi\right) & \text { if } \xi>0 \\ 1 & \text { if } \xi=0\end{cases}
$$

and

$$
\phi_{k}(\xi)=\phi\left(2^{-2 k} \xi\right), \quad k=1,2, \ldots
$$

Obviously $\operatorname{supp} \phi_{0}=[0,4]$ and $\phi_{0}(\xi)=1$ if $\xi \in[0,1]$. Since the kernel of $\phi_{k}(\mathscr{L})$ is $K_{\left(2^{k}\right)}(K$ is the Schwartz kernel of $\phi(\mathscr{L}))$, there exists a constant $C$, which does not depend on $k$, such that for every $f \in L^{p}$,

$$
\begin{gathered}
\left\|\phi_{k}(\mathscr{L}) f\right\| \leqslant C\|f\|, \\
\left\|f-\sum_{j=0}^{k} \phi_{j}(\mathscr{L}) f\right\| \leqslant C\|f\|
\end{gathered}
$$

(for the last inequality we observe that

$$
\left.1-\sum_{j=0}^{k} \phi_{j}(\xi)=\sum_{l=1}^{\infty} \phi\left(2^{-2(k+l)} \xi\right)=1-\phi_{0}\left(2^{-2 k} \xi\right)\right) .
$$

DEFinition. Let $s$ be a nonnegative real number and $1 \leqslant q \leqslant \infty$. The Besov space $B_{p, q}^{s}$ consists of all the functions $f \in L^{p}$ such that

$$
\|f\|_{(s ; p, q)}=\left\|\phi_{0}(\mathscr{L}) f\right\|_{p}+\left(\sum_{k=1}^{\infty}\left(2^{s k}\left\|\phi_{k}(\mathscr{L}) f\right\|_{p}\right)^{q}\right)^{1 / q}<\infty
$$

(obvious modification if $q=\infty$ ).

We consider the operator $\mathscr{L}^{s / 2}=\int_{0}^{\infty} \lambda^{s / 2} d E(\lambda)$. Folland defined in [2] the Sobolev space $H_{p}^{s}$ in a stratified group as the space of all the functions $f \in L^{p}$ such that

$$
\|f\|_{(s, p)}=\|f\|_{p}+\left\|\mathscr{L}^{s / 2} f\right\|_{p}<\infty .
$$

The inclusion relationships between the spaces $B_{p, q}^{s}$ and $H_{p}^{s}$ are a consequence of the following lemma.

Lemma. Let $f \in L^{p}$. Then $\left\|\mathscr{L}^{s / 2} \phi_{k}(\mathscr{L}) f\right\| \leqslant C 2^{s k}\left\|\phi_{k}(\mathscr{L}) f\right\|(k=0,1, \ldots ; s>$ $0)$.

Proof. Let $K^{\prime}$ be the Schwartz kernel of $\mathscr{L}^{s / 2} \phi(\mathscr{L})$. We note that if $k \geqslant 1$, then

$$
\mathscr{L}^{s / 2} \phi_{k}(\mathscr{L})=2^{k s} \int_{0}^{\infty}\left(2^{-2 k} \lambda\right)^{s / 2} \phi\left(2^{-2 k} \lambda\right) d E(\lambda),
$$

and then the kernel of the operator $\mathscr{L}^{s / 2} \phi_{k}(\mathscr{L})$ is $2^{k s} K_{\left(2^{k}\right)}^{\prime}$. Hence,

$$
\begin{aligned}
\left\|\mathscr{L}^{s / 2} \phi_{k}(\mathscr{L}) f\right\| & =\left\|\mathscr{L}^{s / 2}\left(\sum_{j=-1}^{l} \phi_{k-j}(\mathscr{L}) \phi_{k}(\mathscr{L})\right) f\right\| \\
& \leqslant \sum_{j=-1}^{l} 2^{(k-j) s}\left\|\phi_{k}(\mathscr{L}) f * K_{\left(2^{k}\right)}^{\prime}\right\| \leqslant C 2^{k s}\left\|\Phi_{k}(\mathscr{L}) f\right\| .
\end{aligned}
$$


If $k=0$ the same proof works, provided that we observe that the kernel of the operator $\mathscr{L}^{s / 2} \phi_{0}(\mathscr{L})$ is an $L^{1}$ function and that $\phi_{0}(\mathscr{L})=\left(\phi_{0}(\mathscr{L})+\phi_{1}(\mathscr{L})\right) \phi_{0}(\mathscr{L})$. It follows that

$$
\left.\left\|\mathscr{L}^{s / 2} \phi_{k}(\mathscr{L}) f\right\| \leqslant 2^{k s}\left\|\phi_{k}(\mathscr{L}) f\right\| \leqslant\left\|\mathscr{L}^{s / 2} f\right\| \quad \text { (if } f \in L^{p}\right) .
$$

Hence $B_{p, 1}^{s} \subset H_{p}^{s} \subset B_{p, \infty}^{s}$. Moreover, we can prove that $B_{p, q}^{s}$ coincides with the interpolation space $\left(H_{p}^{s_{0}}, H_{p}^{s_{1}}\right)_{\theta, q}$, where $0<\theta<1$ and $s=(1-\theta) s_{0}+\theta s_{1}$ (for the details of the proof, adapt [1, Theorem 6.24]). These results are already contained in Saka's paper [10, Theorem 20 and $\S 6]$.

Let $E_{p}(h, f)$ be the best approximation of a given function $f \in L^{p}$ by means of functions of the space $\mathscr{M}_{p}(h)$; i.e.,

$$
E_{p}(h, f)=\operatorname{Inf}_{g \in \mathscr{M}_{p}(h)}\|g-f\| .
$$

We give a characterization of $B_{p, q}^{s}$ in terms of the best approximation $E_{p}(h, f)$ (see also [13, Theorem 2.5.4 when $G=\mathbf{R}^{n}$ (isotropic)]).

Proposition 2. There exist constants $C_{1}$ and $C_{2}$ such that

$$
C_{1}\|f\|_{(s, p, q)} \leqslant\|f\|_{p}+\left(\sum_{k=0}^{\infty} 2^{k s q} E_{p}\left(2^{k}, f\right)^{q}\right)^{1 / q} \leqslant C_{2}\|f\|_{(s, p, q)}
$$

for every $f \in B_{p, q}^{s}$.

Proof. By Jackson's theorem we have $E_{p}(1, f) \leqslant C\|f\|$. Moreover, the function $\sum_{j=0}^{k-1} \phi_{j}(\mathscr{L}) f \in \mathscr{M}_{p}\left(2^{k}\right)$; hence,

$$
E_{p}\left(2^{k}, f\right) \leqslant\left\|f-\sum_{j=0}^{k-1} \phi_{j}(\mathscr{L}) f\right\| \leqslant \sum_{j=k}^{\infty}\left\|\phi_{j}(\mathscr{L}) f\right\| .
$$

It follows by the Minkowski inequality that

$$
\begin{aligned}
\left(\sum_{k=1}^{\infty} 2^{k s q} E_{p}\left(2^{k}, f\right)^{q}\right)^{1 / q} & \leqslant 2^{s} \sum_{j=0}^{\infty}\left(\sum_{k=0}^{\infty} 2^{k s q}\left\|\phi_{j+k}(\mathscr{L}) f\right\|^{q}\right)^{1 / q} \\
& \leqslant 2^{s} \sum_{j=0}^{\infty} 2^{-j s}\left(\sum_{k=0}^{\infty} 2^{k s q}\left\|\phi_{k}(\mathscr{L}) f\right\|^{q}\right)^{1 / q} .
\end{aligned}
$$

On the other hand, if $\psi \in \mathscr{M}_{p}\left(2^{k-1}\right), k=1,2, \ldots$, then

$$
\phi_{k}(\mathscr{L}) \psi=\phi_{k}(\mathscr{L}) E\left(2^{k-1}\right) \psi=0 \text {. }
$$

Then

$$
\left\|\phi_{k}(\mathscr{L}) f\right\|=\left\|\phi_{k}(\mathscr{L})(f-\psi)\right\| \leqslant C\|f-\psi\| \leqslant C E_{p}\left(2^{k-1}, f\right)
$$

where $C$ does not depend on $k$ (see (6)).

Proposition 3. Let $s$ be a nonnegative real number, and let $k$ and $r$ be two integers such that $k>s-r>0$. Then for every $D \in \mathscr{D}^{r}$ there exists a constant $C$ such that

$$
\left(\int_{0}^{\infty} t^{-q(s-r)-1} \omega_{k}(t, D f)^{q} d t\right)^{1 / q} \leqslant C\left[\left(\sum_{l=0}^{\infty} 2^{l s q} E_{p}\left(2^{l}, f\right)^{q}\right)^{1 / q}+\|f\|\right]
$$

for every $f \in B_{p, q}^{s}$. 
Proof. This proof is a simple adaptation of the proof in $\$ 5.6$ in [9]. Since $\phi_{j}(\mathscr{L}) f \in \mathscr{M}_{p}\left(2^{j+1}\right)$ for every $f \in L^{p}$, it follows by the Bernstein theorem and (8) that

(10) $\left\|D \phi_{j}(\mathscr{L}) f\right\| \leqslant 2^{(j+1) r}\left\|\phi_{j}(\mathscr{L}) f\right\| \leqslant C 2^{j r} E_{p}\left(2^{j-1}, f\right), \quad j=1,2, \ldots$

If $f \in B_{p, q}^{s}$, by Proposition 2 and the Hölder inequality we obtain

$$
\sum_{j=0}^{\infty} 2^{j r} E_{p}\left(2^{j}, f\right) \leqslant\left(\sum_{j=0}^{\infty} 2^{j(r-s) q^{\prime}}\right)^{1 / q^{\prime}}\left(\sum_{j=0}^{\infty} 2^{j s q} E_{p}\left(2^{j}, f\right)^{q}\right)^{1 / q}<\infty .
$$

Hence $D f$ exists (in a distributional sense), and $D f=\sum_{j=0}^{\infty} D \phi_{j}(\mathscr{L}) f$ in $L^{p}$. Corollary 3 (with $N=k$ ) and the Bernstein theorem give

$$
\left\|\Delta_{x}^{k} D \phi_{j}(\mathscr{L}) f\right\| \leqslant C \min \left(2^{j r},|x|^{k} 2^{j(r+k)}\right)\left\|\phi_{j}(\mathscr{L}) f\right\| .
$$

We observe that

$$
\begin{aligned}
\int_{0}^{1} t^{-q(s-r)-1} \omega_{k}(t, D f)^{q} d t & \leqslant C \sum_{N=0}^{\infty} 2^{q(s-r) N} \omega_{k}\left(2^{-N}, f\right)^{q} \\
& \leqslant C^{\prime} \sum_{N=0}^{\infty} 2^{q(s-r) N}\left(\sum_{j=0}^{\infty} 2^{j r} \min \left(1,2^{k(j-N)}\right)\left\|\phi_{j}(\mathscr{L}) f\right\|\right)^{q} .
\end{aligned}
$$

The inequalities (see [9, p. 221])

$$
\sum_{N=0}^{\infty} a^{-q \beta N}\left(\sum_{j=0}^{N} b_{j}\right)^{q} \leqslant C \sum_{j=0}^{\infty} a^{-q \beta j} b_{j}^{q}
$$

and

$$
\sum_{N=0}^{\infty} a^{q \beta N}\left(\sum_{j=N}^{\infty} b_{j}\right)^{q} \leqslant C \sum_{j=0}^{\infty} a^{q \beta j} b_{j}^{q}
$$

(where $a>1$ and $b_{j}>0$ ) allow us to obtain

$$
\left.\begin{array}{l}
\sum_{N=0}^{\infty} 2^{q N(s-r-k)}\left(\sum_{j=0}^{N} 2^{j(r+k)}\left\|\phi_{j}(\mathscr{L}) f\right\|\right)^{q}, \\
\sum_{N=0}^{\infty} 2^{q(s-r) N}\left(\sum_{j=N}^{\infty} 2^{r j}\left\|\phi_{j}(\mathscr{L}) f\right\|\right)^{q}
\end{array}\right\} \leqslant C \sum_{j=0}^{\infty} 2^{s q j}\left\|\phi_{j}(\mathscr{L}) f\right\|^{q} .
$$

Finally by (12), (13) and (8) we have

$$
\begin{aligned}
\left(\int_{0}^{1} t^{-q(s-r)-1} \omega_{k}(t, D f)^{q} d t\right)^{1 / q} & \leqslant C\left(\sum_{j=0}^{\infty} 2^{s q j}\left\|\phi_{j}(\mathscr{L}) f\right\|^{q}\right)^{1 / q} \\
& \leqslant C\left(\|f\|+\left(\sum_{j=0}^{\infty} E_{p}\left(2^{j}, f\right)^{q}\right)^{1 / q}\right) .
\end{aligned}
$$


Moreover, it follows by (10) that

$$
\begin{aligned}
\int_{1}^{\infty} t^{-q(s-r)-1} \omega_{k}(t, D f)^{q} d t & \leqslant C\|D f\|^{q} \int_{1}^{\infty} t^{-q(s-r)-1} d t \\
& \leqslant C\left(\|f\|+\left(\sum_{j=0}^{\infty} 2^{s q j} E_{p}\left(2^{j}, f\right)^{q}\right)^{1 / q}\right) .
\end{aligned}
$$

Proposition 4. Let $s$ be a nonnegative real number, and let $k$ and $r$ be integers such that $k>s-r>0$. The norms

$$
\begin{aligned}
& \|f\|_{(*)}\|f\|+\left(\int_{0}^{\infty} t^{-q(s-r)-1} \omega_{k}\left(t, f^{(r)}\right)^{q} d t\right)^{1 / q}, \\
& \|f\|_{(* *)}=\|f\|+\left(\int_{0}^{\infty} t^{s q-1} E_{p}(t, f)^{q} d t\right)^{1 / q}
\end{aligned}
$$

are equivalent to the norm $\|f\|_{(s, p, q)}$.

Proof. It follows by the previous propositions that it is enough to prove $\|f\|_{(* *)} \leqslant C\|f\|_{(*)}$. By (5) and Corollaries 2 and 3 we obtain

$$
\begin{aligned}
E_{p}(t, f) & \leqslant \int_{G}|K(v)|\left\|\Delta_{\delta_{1 / 1} v^{-1}}^{N} f\right\| d v \\
& \leqslant C t^{-r} \int_{G}|K(v) \| v|^{r} \omega_{N-r}\left(\frac{|x|}{t}, f^{(r)}\right) d v \\
& \leqslant C t^{-r} \omega_{N-r}\left(\frac{1}{t}, f^{(r)}\right) \int_{G}|K(v) \| v|^{r}(1+|v|)^{N-r} d v
\end{aligned}
$$

Hence,

$$
\int_{0}^{\infty} t^{s q-1} E_{p}(t, f)^{q} d t \leqslant C \int_{0}^{\infty} t^{(s-r) q-1} \omega_{N-r}^{q}\left(\frac{1}{t}, f^{(r)}\right) d t
$$

\section{REFERENCES}

1. J. Bergh and J. Löfström, Interpolation spaces, Springer-Verlag, Berlin, 1976.

2. G. B. Folland, Subelliptic estimates and function spaces on nilpotent Lie groups, Ark. Mat. 13 (1975), $161-207$.

3. Lipschitz classes and Poisson integrals on stratified groups, Studia Math. 66 (1979), 37-55.

4. G. B. Folland and E. M. Stein, Hardy spaces on homogeneous groups, Math. Notes 28, Princeton Univ. Press, Princeton, N. J., 1982.

5. S. Giulini, Bernstein and Jackson theorems for the Heisenberg group, J. Austral. Math. Soc. Ser. A. 38 (1985), 241-254.

6. I. R. Inglis, Bernstein's theorem and the Fourier algebra of the Heisenberg group, Boll. Un. Mat. Ital. A (6) 2 (1983), 39-46.

7. S. G. Krantz, Lipschitz spaces on stratified groups, Trans. Amer. Math. Soc. 269 (1982), 39-66.

8. G. C. Mauceri, Multipliers, maximal operators and Riesz means on stratified groups, Symposia Math. (to appear). 
9. S. M. Nikol'skii, Approximation of functions of several variables and imbedding theorems, SpringerVerlag, Berlin, 1975.

10. K. Saka, Besov spaces and Sobolev spaces on a nilpotent Lie group, Tôhoku Math. J. (2) 31 (1979), 383-427.

11. P. M. Soardi, On nonisotropic Lipschitz spaces, Harmonic Analysis, Lecture Notes in Math., vol. 992, Springer-Verlag, Berlin, 1983.

12. _ Lipschitz spaces on homogeneous groups, preprint.

13. H. Triebel, Interpolation theory, function spaces, differential operators, North-Holland, Amsterdam, 1978.

Dipartimento di Matematica, Universita di Milano, via Saldini 50, 20133 Milano, Italy 